\title{
A Personalized Music Recommendation Algorithm Based on User Implicit Feedback and Weighted Socialized Tag Content Filtering
}

\author{
Yang Yang ${ }^{1, a, *}$, Wang Qiang ${ }^{1, b}$ \\ School of Economics and Management, Beihang University, Xueyuan Road, Beijing,China

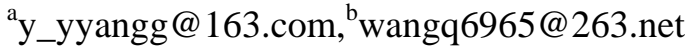 \\ *Yang Yang
}

Keywords: Personalized Recommendation, Implicit Feedback, Socialized Tag, Content Filtering

\begin{abstract}
The popularity of the Internet and the electronization of music resources make it easier for people to get music what they like. However, facing abundant resources, it is difficult for people to find their favorite music accurately and timely. Personalized music recommendation algorithms play an increasingly important role in online music service systems. Traditional feedback method requires the user to give feedback explicitly, which not only increases information collection cost but also gives the user an additional burden. This paper proposes a content filtering recommendation method based on user implicit feedback and weighted socialized tags, and designs experiment to compare our recommendation method with fuzzy theory method. The experimental results show this method is more accurate than the fuzzy theory method in recommending music to the user.
\end{abstract}

\section{Introduction}

With the rapid development of the Internet and the wide implementation of music resources electronization, people can easily find a variety of music resources in a huge music electronic library. The music consumption patterns become different. People used to buy records or use music website to get songs. Now people can listen to, upload and share music through the online music service system $^{[1]}$. Facing massive music data, if the user cannot easily find their favorite music, it will extremely affect their experience. Thus, an effective personalized music recommendation algorithm is particularly important for the online music service system. Traditional relevance feedback method requires the user to give explicit feedback, such as scoring, specifying keywords, selecting and tagging files, and answering questions ${ }^{[2]}$. This feedback method enforces the user to do something beyond its normal activities, which increases the cost of information collection and gives the user an additional burden. Instead, the implicit feedback technique only collects natural interactive information between the user and the system, for example, browsing, listening or collection. It not only has a little intervention in user's behavior, but collect a lot of information as well. So taking advantade of implicit feedback information and combining it with the personalized recommendation have practical significance. In this paper, we proposes a content filtering recommendation method based on user implicit feedback and weighted socialized tag for online music system.

\section{Related Research}

\subsection{User Feedback}

User behavior can be captured by online systems when they browse the site in the form of data. These data reflects the users' real interest to some extent. We refer to it as user feedback, which is classified into explicit feedback and implicit feedback ${ }^{[3]}$ according to the accuracy of the feedback.

Explicit feedback, which clearly reflects the user preferences. For example, upvoting, downvoting, scoring and so on. There are two general categories of explicit feedback: quantitative explicit feedback and dichotomous explicit feedback. 10 points or 5 points scoring system reflects 
the degree of user performance for items. Dichotomous explicit feedback includes: "top" and "step", "heart" and "trash", which represents like and dislike respectively and no dinference in degree of like and dislike.

Implicit feedback cannot clearly reflect the behavior of the user preferences. In terms of listening to music, clicking on the "next song", labeling the song, the order and the frequency of songs played, all these behaviors reflect the user's implicit feedback. Although these behaviors cannot explicitly represent the user's point of view, it is possible to infer the user's preferences by analyzing these information. For example, a song repeatedly appears in the user's play records, you can speculate that he may like this song; on the contrary, a song which is skipped by the user when he just listen to the half of it could be less welcomed for this user.

\subsection{Socialized Tag}

Socialized tags, which are often used to describe and ccategorize an entity, a concept or a perspective, are generated spontaneously by users of social platforms in the form of phrases or keywords ${ }^{[4]}$. Because users use their own language to produce them, the tags have the following characteristics: 1) the semantics meaning of tags indicate the user's understanding and feelings of the entity ${ }^{[5]}$. 2) different users may label the same resource differently. Becuase different individuals have different perception of music. 3) the most frequent tags of music entities reflect objective information. Although the individual's perception of music is different, the most frequenty tag is the result drawn from all the user experiences in the social platform, which most people agree on. Therefore, the most frequent label represents the entire community's view of the music entity, which is close to the objective information of the entity ${ }^{[6]}$. 4) tags, composed of natural language such as sentences or words, have good semantic interpretation. They are easier for public to understand than acoustic features (pitch, rhythm, melody, etc.).

\section{Method}

This paper proposes a music recommendation algorithm based on the user's implicit feedback information and weighted socialized tags to filter content. This method mainly includes three modules: user behavior analysis, user interest modeling and filtering recommendation. Figure 1 shows the overall flow diagram of the recommendation system.

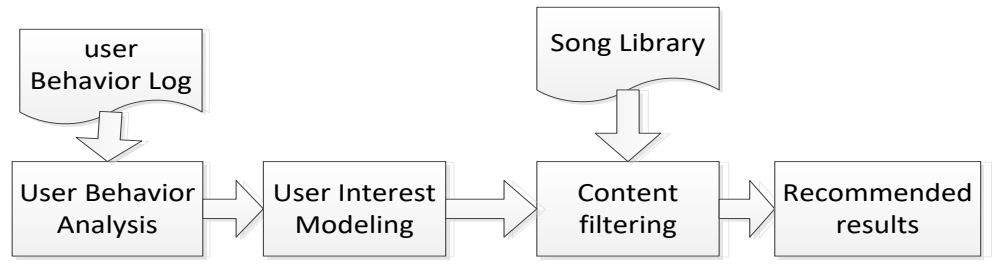

Figure 1 flow diagram of recommendation system

First of all, the system detects the user's feedback (skip, normal play and repeat) by analyzing the sequence of the user logs, and divides the songs into three groups (skip set, normal set and repeat set) according to the user behavior. Then we collect the most frequent tags and assign weights for the tags according to the frequency of the tags in the collection. Finally, the implementation of the content filtering method is used to get recommended results for the user. Experiments show that this method which fully-considered user behavior can more accurately describe the user's interest, making the recommended results better.

\subsection{User Behavior Modeling}

In order to obtain the user's preference, this article analyzes the user log to obtain the user's implicit feedback information. The method of this article is based on a simple hypothesis: the user playing single song cycle shows that the user loves the song; user who often skips a song shows he does not like the song, although once skip cannot infer that, but skip many times can make the probability of the user does not like the song relatively large. 
The user can control the playing of the music player through the "next song" or "last song" button in the actual use of the online music service system. The log will record the start time of each song and the id of the song. The resulting log can be formalized as follows when a user in the use of online music service system during a session,:

$$
\text { Session: }\left\{<\mathrm{MBID}_{1}, \mathrm{t}_{1}>,<\mathrm{MBID}_{2}, \mathrm{t}_{2}>,<\mathrm{MBID}_{3}, \mathrm{t}_{3}>\ldots<\mathrm{MBID}_{\mathrm{n}}, \mathrm{t}_{\mathrm{n}}>\right\}
$$

$M B I D$ is the unique identifier of the song in the log, and $t$ is the timestamp when the song starts playing.

Based on the above assumptions about user behavior and the form of user logs, the user log is analyzed as follows:

First step, calculate the playing time of each song, because the log records are timestamp, so the song playback time is: $\Delta\left(t_{n}, t_{n+1}\right)=t_{n}-t_{n+1}$.

Second step, compare the song's playing time and its original length, if the user clicks on the "next song" button, the playing time is longer than the original length.

Last step, compare the MBID of the adjacent records in log, if the user clicks on the "previous song" button, then the MBID of adjacent records is the same.

Then formalize user behavior as follows:

Skip. In a session, If the user played a song did not finished, we judge the user clicked on the "next song" button, then we mark the song as "Skip". Formalized as follows, $S$ is the threshold for determining whether or not a skip behavior, this article assumes that the user skip the song which he listen to $50 \%$ of the original length. That means $S=50 \%$.

$$
\operatorname{Skip}\left(\mathrm{l}_{\mathrm{n}}, \mathrm{l}_{\mathrm{n}+1}\right)= \begin{cases}\text { true } & \text { if } \Delta\left(\mathrm{t}_{\mathrm{n}}, \mathrm{t}_{\mathrm{n}+1}\right)<S \\ \text { false } & \text { if } \Delta\left(\mathrm{t}_{\mathrm{n}}, \mathrm{t}_{\mathrm{n}+1}\right) \geq \mathrm{S}\end{cases}
$$

Repeat. In a session, If the user repeatedly played a song, we judge the user clicked on the "last song", we mark the song as "Repeat".

$$
\text { Compare }\left(l_{n}, l_{n+1}\right)=\left\{\begin{array}{cc}
\text { true } & \text { if } l_{m} \text { equals } l_{n+1} \\
\text { false } & \text { otherwise }
\end{array}\right.
$$

Normal. In a session, the user only play a song once, at this time the user normally listened to the song and did not make the operation, marked as "Normal".

\subsection{User Interest Modeling}

Through the analysis of user behavior in the previous section, we got the skip set, repeat set and normal set, the difference between the collections shows the difference in user interest. We analysis the user behavior of the three song sets, combine them with weighted social tags, establish a user interest model.

First, replace the content of song with the most frequent social tags of the song in the three song sets, and get the three tag sets; then remove the duplicate parts of the tag set and assign the weight to the frequency of the tag in the respective set. Then we get three tag vector positive tags (PT), Normal tags (NMT), and Negative tags (UT). Finally the user interest model is obtained by linearly combining the three tag vectors $(\alpha, \beta, \gamma$ for the mixed parameter). The label weight is calculated as follows:

$$
\operatorname{Score}\left(t_{i} \mid u\right)=\alpha \times \operatorname{score}\left(t_{i} \mid u, P T\right)+\beta \times \operatorname{score}\left(t_{i} \mid u, N M T\right)+\gamma \times \operatorname{score}\left(t_{i} \mid u, U T\right)
$$

Where the Score $\left(t_{i} \mid u\right)$ is the frequency of the tag $t_{i}$ in the positive tags of the user $u$ and $0<\alpha<1,0<\gamma \leq \beta<1$.

The key part of content filtering is to project the user interest model and the item feature vector into the same vector space. Therefore, it is necessary to project the song into the label space. This paper uses the most frequent social tag set of the song as the feature set of the song. The most frequent social tag collection of candidate song $i$ is marked as iT, then the user's preference for the song is predicted as follows:

$$
r_{u i}=\alpha \times \sum_{t_{i} \in \text { iTnPT }} \operatorname{score}\left(t_{i} \mid u, P T\right)+\beta \times \sum_{t_{i} \in \text { iTกNMT }} \operatorname{score}\left(t_{i} \mid u, N M T\right)+\gamma \times \sum_{t_{i} \in \text { iTnNT }} \operatorname{score}\left(t_{i} \mid u, N T\right)
$$


Where $\sum_{\mathrm{t}_{\mathrm{i}} \in \mathrm{iT \cap PT}} \operatorname{score}\left(\mathrm{t}_{\mathrm{i}} \mid \mathrm{u}, \mathrm{PT}\right)$ is the sum of the weights of the common tags in iT and PT, and this value is regarded as the degree to which the user likes the song. Similarly, $\sum_{\mathrm{t}_{\mathrm{i}} \in \mathrm{iTnNMT}} \operatorname{score}\left(\mathrm{t}_{\mathrm{i}} \mid \mathrm{u}, \mathrm{NMT}\right)$ represent that the user does not like nor hate the song, $\sum_{\mathrm{t}_{\mathrm{i}} \in \mathrm{iT \cap NT}} \operatorname{score}\left(\mathrm{t}_{\mathrm{i}} \mid \mathrm{u}, \mathrm{NT}\right)$ indicates that the user does not like the degree of the song.

\section{Experiments and Results}

The dataset used in this paper is the public data set (Last.fmDataset-1Kusers) provided by the large music social website Last.fm. The data set contains 992 users during the period of May 2009 to May 2010, the total number of records is $19,150,868$, because the data set does not contain the required method of the song social label (top tags and duration, so all of this information is retrieved through the web crawler through the track.info provided by Last.fm and added accordingly to the original dataset. The data set belongs to the same user and the time of the nearest 1/3 record assigned to the test set, the remaining $2 / 3$ for the training set. In the user behavior analysis model, the effective interval for session is 1 hour, that is, if the time difference between the two records adjacent to the same user is greater than 1 hour, they are not in the same session.

In order to verify the validity of the tags with weight, this paper will be compared with the fuzzy theory of content filtering method without the weighted socialized tags proposed in paper ${ }^{[8]}$. The three mixed parameters in the user interest model are adjusted by parameter adjustment: $\alpha=1, \beta=$ $-1, \gamma=0.5$.

The evaluation index of this experiment is as follows, where $k$ is the recommended list length.

1) Precision

$$
\text { Precision }=\frac{\sum_{\mathrm{u} \in \mathrm{U}}\left|\mathrm{R}_{\mathrm{k}}(\mathrm{u}) \cap \mathrm{T}(\mathrm{u})\right|}{\sum_{\mathrm{u} \in \mathrm{U}}\left|\mathrm{R}_{\mathrm{k}}(\mathrm{u})\right|}
$$

2) Recall

$$
\text { Recall }=\frac{\sum_{\mathrm{u} \in \mathrm{U}}\left|\mathrm{R}_{\mathrm{k}}(\mathrm{u}) \cap \mathrm{T}(\mathrm{u})\right|}{\sum_{\mathrm{u} \in \mathrm{U}}|\mathrm{T}(\mathrm{u})|}
$$

The precision of the algorithm is shown in Figure 2

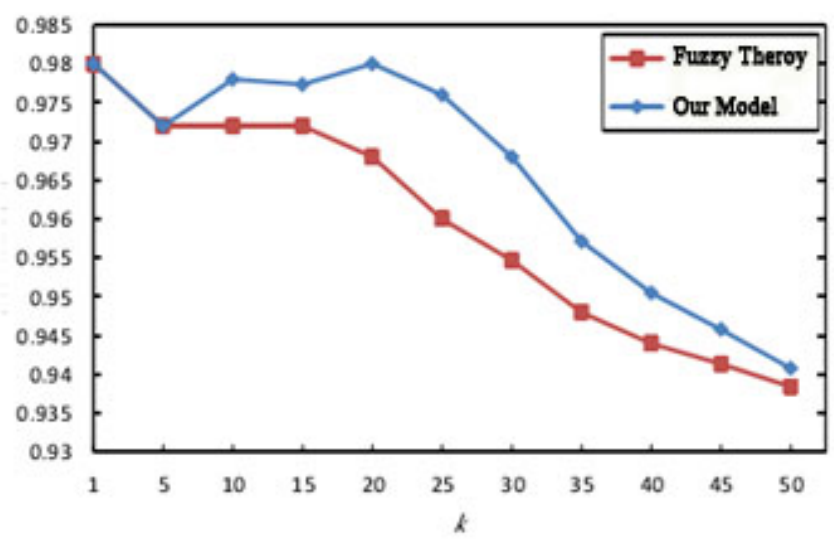

Figure 2 The precision of the algorithm

As what can be seen from Figure 2, the recommended precision is declining as the length of the recommended list increases. The method is better than fuzzy method in precision. When the value of $\mathrm{k}$ is 1 to 5 , the precision is no significant difference, but when the value is greater than 5 , the precision difference is obvious. When $\mathrm{k}$ is 25 , the difference is the largest, and the precision of this method is 0.976 , Fuzzy method precision is only 0.96 , the average increasing of precision is $1.68 \%$.

In Figure 3, with the recommended list increase, the recommended recall rate becomes larger. This paper is slightly better than the fuzzy method in the recall rate index.

Compared with the experimental results, the content filtering method based on the weighted socialized tags is superior to the fuzzy theory in terms of precision and recall rate. This is due to the user interest model with the weighted tag, which can better explain what the user likes and does not 
like, because higher weight of the tag implies the user's preferred message, and vice versa.

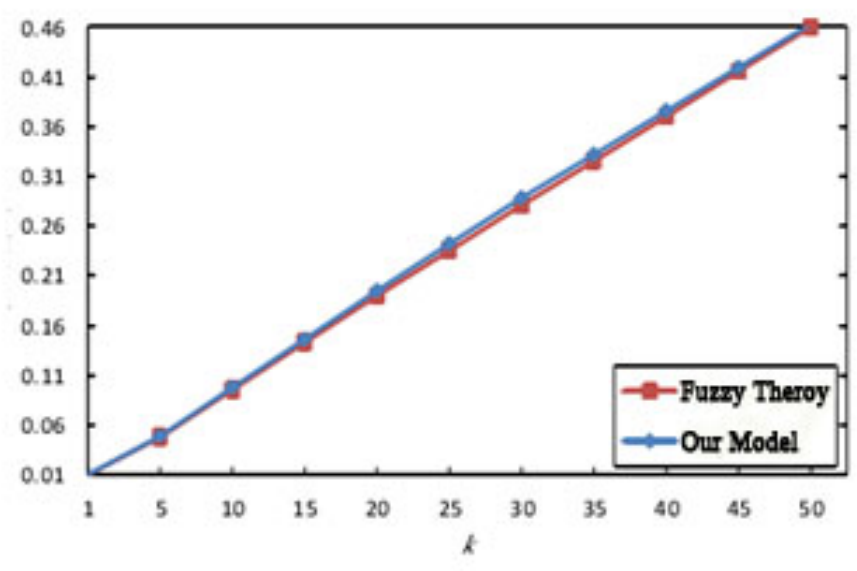

Figure 3 The Recall rate of the algorithm

\section{Conclusion}

The user's behavior contains a lot of user implicit feedback information. These actions can effectively reflect the user's preferences for different items. Based on the analysis of user behavior, this paper proposes a content filtering method with weighted socialized tags based on user implicit feedback. The experiment shows that the modeled behavior of user and the weight of social tags can be better guess the user's preferences in music Recommendations.

\section{References}

[1] China Internet Network Information Center. 2014 China Social Application User Behavior Research[EB/OL].[2014-08-22].http://www.cnnic.cn/hlwfzyj/hlwxzbg/201408/t20140822_47862.ht m.

[2] North A, Hargreaves D. The social and applied psychology of music[M]. Oxford University Press, 2008.

[3] Diane K, Jaime T. Implicit feedback for inferring user preference: a bibliography[J]. SIGIR Forum, 2003, 2(37): 18-28.

[4] Selamat A, Thanh N.N., Haron H, et al. A Semantically Enhanced Tag-Based Music Recommendation Using Emotion Ontology[C]. ACIIDS 2013, Springer Berlin Heidelberg, 2013:119-128.

[5] Jeng S.P., Shyi M.C., Thanh N.N., et al. Generation of Tag-Based User Profiles for Clustering Users in a Social Music Site[C]. $4^{\text {th }}$ Asian Conference, ACIIDS 2012, Kaohsiung, Taiwan, Springer Berlin Heidelberg, 2012: 51-61.

[6] Roberto B, Fabrizio S.G.S et al.Myusic: a Content-based Music Recommender System based on eVSM and Social Media[C]. Proceedings of the 4th Italian Information Retrieval Workshop, Pisa,Italy, 2013:65-72.

[7] Dimitrios R, Apostolos A, Jonas E, et al. Content-based tag propagation and tensor factorization for personalized item recommendation based on social tagging[J]. ACM Transactions on Interactive Intelligent Systems, 2014, 4(3): 1-27.

[8] Klaas B, Elias P, Etienne E K. Evaluating and Analysing Dynamic Playlist Generation Heuristics Using Radio Logs and Fuzzy Set Theory[C]. The 10th International Conference on Music Information Retrieval (ISMIR), 2009: 351-356. 